Machine Learning 2: 195198,1987

(C) 1987 Kluwer Academic Publishers. Boston - Manufactured in The Netherlands

\title{
EDITORIAL
}

\section{Research Papers in Machine Learning}

\section{A prototype for machine learning papers}

Scientists do not work in isolation. Communication is central to the scientific enterprise, and much of the exchange occurs through research papers. Naturally, the content of these papers will vary with the field of study, and in this essay I discuss the information that machine learning researchers should attempt to communicate in their articles. Readers may interpret these comments as reflecting the current editorial policy of the journal Machine Learning. though this policy will undoubtedly evolve over time, as even editors and editorial boards learn from experience.

Despite the advantages of standardization, it can lead a new discipline to crystallize into a rigid pattern before its time. Rather than define an inflexible format that all papers in machine learning must follow. I provide below a more flexible, prototypical outline. Specific papers will diverge from this prototype, but it should provide a useful target, particularly for authors now to the field. The outline assumes a paper that describes a single, rumning machine learning system. Most papers that appear in Machine Learning will take this form, but later I will briefly consider some other possibilitics.

1. Goals of the research. Machine learning researchers have many different reasons for carrying out their work. Some are interested in general principles of intelligent behavior, others are concerned with modeling human learning. and still others are oriented towards applications. If an author hopes to communicate with his audience, it is essential that he state his goals early in the paper. so that readers can decide for themselves whether the work has made progress towards those goals.

2. Description of the task. Learning always occurs in the context of some performance task such as classification. planning. or language understanding and, for a given performance task, different learning tasks are possible. For instance, learning from examples and conceptual clustering both involve classification. but they differ in the information given and in the nature of the learned structures. The author should clearly state both the performance and learning tasks he is studying. specifying the given inputs and the desired outputs. 
3. Representation. Any learning system must represent its inputs - the data and knowledge on which learning is based - and its outputs - the knowledge it acquires. The author should describe the representational scheme used for both types of information.

4. Performance component. Learning involves some improvement in performance, and the author should describe the system's performance element in enough detail to support description of the learning method.

5. Learning algorithms. Naturally, the learning method should be a central focus of any paper on machine learning. The author should clearly describe the basic steps of the learning mechanism under study, using flow charts or English paraphrases of the algorithm to supplement the text. Ideally, he should describe the learning method in sufficient detail to let readers reconstruct the system on their own. The ability to replicate results is essential to any true science.

6. Detailed examples. No matter how clear the abstract description of a learning algorithm, it can always be clarified by examples. The author should include at least one detailed example of his method in operation. Figures accompanied by text usually communicate such examples far better than do program traces.

7. Evaluation. Without some evaluation of the learning method, readers cannot know whether the work constitutes a step forwards or backwards. This section is central to any machine learning paper. Of course, one can imagine a variety of evaluation schemes, differing according to the author's goals. The most obvious alternatives are:

- Empirical evaluation. This approach tests the learning algorithm's behavior empirically under varying conditions and using different metrics. For instance, one might show that the time taken to converge on useful structures degrades with increases in noise.

- Theoretical evaluation. This approach proves theorems about the behavior of learning algorithms. For example, one might show that the number of instances needed to acquire the correct structure increases as a polynomial function of the structure's complexity.

- Psychological evaluation. This approach compares the algorithm's behavior to human learning behavior. For example, a given model might explain the power law of practice, a phenomenon that has been observed in human skill acquisition across many domains.

Note that these methods are not contradictory; one can evaluate a single method along all three dimensions. One can also imagine other evaluation schemes, and the field has plenty of room for alternative paradigms; however, all such paradigms should be based on some principled evaluation techniques. The issue of generality cuts across all such paradigms, and authors should attempt to characterize the class of tasks for which their results hold. 
8. Related work. Ideas never occur in a vacuum, and the methods described in a paper will invariably bear an interesting relation to earlier research. The author should describe how his approach differs from its predecessors and how it is similar. He should also point out the contribution of both the earlier and the current work.

9. Future research. Science is a never-ending process, and no matter how impressive a given piece of research, there is always more work to be done. The author should clearly state the limits of his approach to learning and list the questions he has failed to answer. He should then outline his plans for remedying these drawbacks in future efforts.

The order of these sections is less important than their content, though clearly some should precede others. The author need not divide the material in precisely this fashion, provided he presents the information in some manner. But the above outline should serve as a useful prototype for research papers on machine learning.

\section{Machine learning as an empirical science}

One of the evaluation techniques mentioned above involved the empirical study of an algorithm's behavior, and this approach is prevalent enough within machine learning to deserve further discussion. Most sciences are empirical in nature, and this means their theories are based on robust empirical phenomena, such as the law of combining volumes and the ideal gas law. A growing number of machine learning researchers are focusing their efforts on discovering analogous phenomena in the behavior of learning systems, and this is an encouraging sign.

In many sciences, such phenomena are stated in terms of relations between independent and dependent variables. ${ }^{1}$ In machine learning, two natural independent terms are the structure of the knowledge to be learned and the regularity in the environment (e.g., the amount of noise in the data). For incremental learning methods. one may also vary the order in which data are presented and the stability of the environment over time. Most natural dependent measures are related to some performance criterion. such as diagnostic accuracy (for classification tasks) or quality of solution paths (for problem-solving tasks). However, these are not the only such variables, and one important task awaiting machine learning researchers is the identification of relevant independent terms and useful metrics.

Recent work along these lines has been carried out by Quinlan (1986), Fisher (1987), and others. Although much of the experimental work has examined the behavior of empirical learning methods, it can also be applied to explanation-based techniques. and O'Rorke (1987) reports a set

\footnotetext{
${ }^{1}$ Of course, simply collecting data is not enough; empirical results are useful only to the extent that thev load to deener understanding
} 
of experiments within this framework. In other words, the experimental evaluation of learning algorithms is a general approach that can be usefully applied to all machine learning paradigms.

\section{Other types of machine learning papers}

Papers that diverge from the prototype outlined above may still contribute to the field. For instance, articles that compare the empirical behavior of different algorithms (e.g., Schlimmer \& Fisher, 1986) will necessarily spend less time describing the methods themselves and more time discussing their behavior. Purely theoretical papers (e.g., Rivest, 1987) may focus on task characteristics that hold for all algorithms, and thus devote their discussion to statements and proofs of theorems.

Syntheses of earlier results also have a role to play, since they can reveal underlying connections between tasks and methods that were previously thought disparate. Such papers need not present any new empirical results, but they will prove more useful to the extent that they suggest new variations on tasks and methods. Naturally, the threshold for accepting survey papers should be higher than that for alternative formats.

Machine learning is an evolving discipline, and the nature of its research papers must change along with the interests of its constituents. But hopefully, the formats and evaluation criteria outlined above will serve the field well for some years to come, as we explore the empirical, theoretical, and psychological facets of learning.

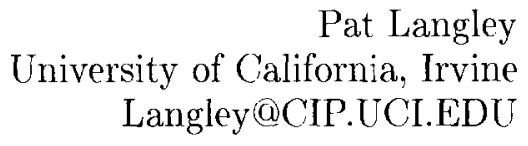

\section{References}

Fisher, D. H. (1987). Knowledge acquisition via incremental conceptual clustering. Machine Learning, 2, 139-172.

O'Rorke, P. (1987). LT revisited: Experimental results of applying explanationbased learning to the logic of Principia Mathematica. In Proceedings of the Fourth International Workshop on Machine Learning (pp. 148-159). Irvine, CA: Morgan Kaufmann.

Quinlan, J. R. (1986). Induction of decision trees. Machine Learning, 1, 81-106. Rivest, R. L. (1987). Learning decision lists. Machine Learning, 2, 229-246.

Schlimmer, J. C.. \& Fisher, D. H. (1986). A case study of incremental concept induction. Proceedings of the Fifth National Conserence on Artificial Intelligence (pp. 496-501). Philadelphia, PA: Morgan Kaufmann. 